\title{
Spatial Site-Patterning of Wettability in a Microcapillary Tube
}

\author{
Yali Zhang, ${ }^{\dagger}$ Jan W. van Nieuwkasteele, ${ }^{\dagger}$ Meng Qiang, ${ }^{\ddagger}$ Peichun Amy Tsai, ${ }^{\text {II }}$ and Rob G.H. Lammertink ${ }^{*}{ }^{\dagger}$ \\ ${ }^{\dagger}$ Soft Matter, Fluidics and Interfaces, MESA+ Institute for Nanotechnology, Faculty of Science and Technology, University of \\ Twente, Enschede, The Netherlands \\ ${ }^{\ddagger}$ Mechanical Engineering, University of Science and Technology Beijing, Beijing, China \\ ${ }^{\mathrm{I}}$ Department of Mechanical Engineering, University of Alberta, Edmonton, Alberta, Canada
}

\section{Supporting Information}

ABSTRACT: Substrate functionalization is of great importance in successfully manipulating flows and liquid interfaces in microdevices. Herein, we propose an alternative approach for spatial patterning of wettability in a microcapillary tube. The method combines a photolithography process with self-assembled monolayer formation. The modified microcapillaries show very sharp boundaries between the alternating hydrophilic/hydrophobic segments with an achieved smallest domain dimension down to $60 \mu \mathrm{m}$ inside a $580 \mu \mathrm{m}$ inner diameter capillary. Our twostep method allows us to pattern multiple types of functional groups in an enclosed channel. Such structures are promising regarding the manipulation of segmented flows inside capillaries.

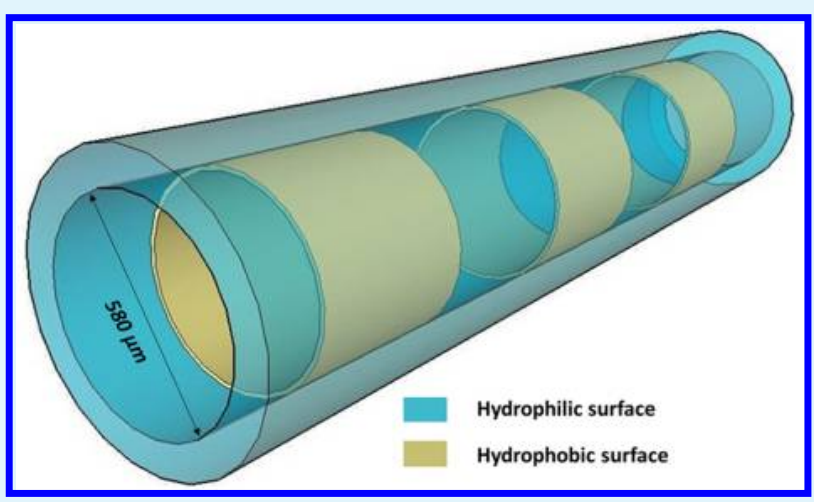

KEYWORDS: surface modification, patterning, micro channel, wetting

S ubstrate functionalization plays a key role in manipulating the usage of microsystems in biomedical/chemical analysis. Selectively tuning of surface wettability facilitates new applications for microsystems and promotes fundamental understanding, for example, in manipulating droplets, ${ }^{1,2}$ creating hydrophobic valves, ${ }^{3,4}$ solvent extraction of metal ions or organics, ${ }^{5,6}$ and attaching biomaterials. ${ }^{7,8}$ Patterning dissimilar surface chemistries on flat substrates is generally accomplished using techniques such as microcontact printing, electrochemical deposition, and self-assembling monolayer (SAM) formation combined with photomasks. ${ }^{9-12}$ Techniques to modify surfaces in enclosed microchannels are often complicated and have their limitations. Soft photolithography process (e.g., microcontact printing) are generally suitable for patterning flat substrates. ${ }^{8,13}$ Local heat induced patterning by particles $^{14}$ causes significant thickness difference $(>\mu \mathrm{m})$ between the hydrophilic and hydrophobic regions. Electrochemical deposition, ${ }^{15}$ often applied to microchip-based systems, requires complex chip design and implemented electrodes. Vong ${ }^{16}$ has fabricated dissimilar wettability in a microchannel by using local photochemical reaction with a tailored linker in a complex route. Photochemical patterning in enclosed channels is also demonstrated by decomposing or chemical modifying the coated monolayers under UV irradiation by using either photocatalysts ${ }^{17,18}$ or UV photochemistry. ${ }^{19}$ Such methods require certain photoresponsive materials and chemistries. The UV photochemistry method presented by Chen et al. ${ }^{19}$ is particularly useful to pattern multiple monolayers within enclosed channels. Special care must be taken to prevent the resulting hydrophilic regions to turn hydrophobic after several days. ${ }^{20}$ Flow controlled surface modification is capable of fabricating defined heterogeneous wettability inside a microchannel but is restricted by the fluid flow pattern. ${ }^{21}$

In this communication, we propose an alternative approach for spatial patterning inside a microcapillary tube. Alternating hydrophilic/hydrophobic segments were obtained by combining photolithography with silane based self-assembling monolayer chemistry. The photolithography process defines the boundary of hydrophilic/hydrophobic regions by patterning a dense photoresist layer on the targeted hydrophilic regions. The coated layer is dense and resistant enough to protect the covered surfaces from the subsequent silane monolayer formation step. We further demonstrate the effect of these patterns inside the capillaries on the fluid dynamics of segmented liquid-liquid flows.

As outlined in Figure 1, a dense positive photoresist layer (Olin Oir 907, a novolac resin) was coated on the inner wall of a hydrophilic microcapillary. We defined the boundary between the hydrophilic/hydrophobic segments by exposing the photoresist-coated microcapillary to parallel UV light $(250-350 \mathrm{~nm}$, $12 \mathrm{~mW} / \mathrm{cm}^{2}$ ) through a photomask with a desired pattern. The UV-exposed photoresist layer was removed using a developer solution to expose the targeted surfaces and dried. The wettability of these exposed surfaces was modified by exposure to an organosilane solution. Finally, we exposed the hydrophilic

Received: February 12, 2016

Accepted: April 15, 2016

Published: April 15, 2016 


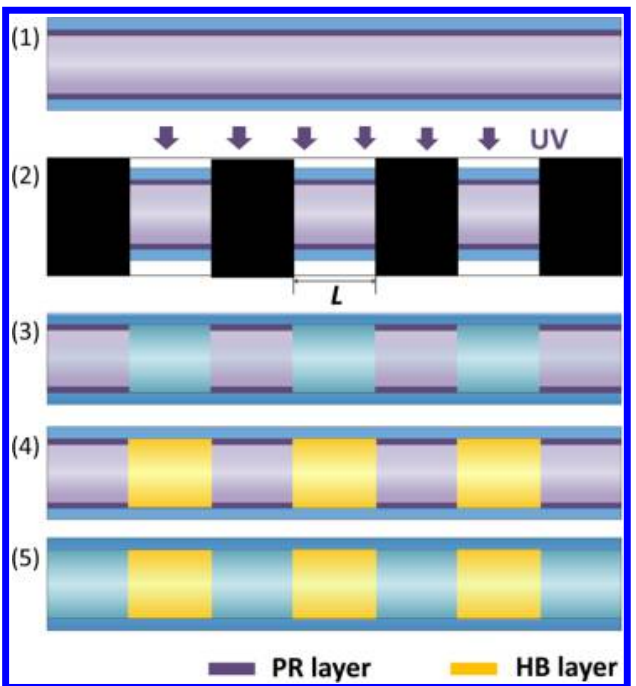

Figure 1. Schematic drawing of the fabrication procedure regarding heterogeneous patterning of surface wettabilities. HB-hydrophobic. (1) Coating a positive photoresist layer on the inner capillary wall. (2) UV exposure under a patterned mask. (3) Removing the exposed positive photoresist layer using developer. (4) Hydrophobizing the exposed surface with a monolayer of silane coating. (5) Removing the photoresist layer.

regions by flowing dehydrated hexane to remove the silane residues followed by a mixture of acetone and ethanol $(1: 1 \mathrm{v} / \mathrm{v})$ to remove the photoresist layer.

We verified the feasibility of this approach using flat glass slides. The static water contact angles were measured to be 28 $\pm 3^{\circ}$ and $108 \pm 3^{\circ}$ on the hydrophilic and hydrophobic surfaces, respectively (see Figure 2). This demonstrates that the patterned photoresist layer acts as a protective layer and also keeps these surfaces relatively hydrophilic after it is removed. This allows to apply an optional second surface modification afterward. Careful cleaning is essential to remove all the unreacted silane before removing the photoresist layers, to ensure that the subsequent exposed hydrophilic surfaces stay intact. Atomic force microscopy (AFM) measurements demonstrate that there were no large aggregates and defects on the modified surfaces. The boundary between the modified and unmodified surfaces is very sharp. The formed SAM layer is uniform with a measured thickness of $\sim 1 \mathrm{~nm}$, and an surface average roughness less than $0.66 \mathrm{~nm}$, which is only slightly larger compared to the uncoated glass surface. We have applied various silane chemistries for the hydrophobization process via both liquid and gas phase reactions. Different surface wettabilities were obtained, represented by water contact angles $\left(2 \mu \mathrm{L}\right.$ per droplet) of $75 \pm 2^{\circ}$ for chlorotrimethylsilane (CTMS, via gas phase deposition) and $100 \pm 4^{\circ}$ for octyltriethoxysilane (OTES, via liquid solution deposition) modified surfaces, respectively, and very low contact angles for all the hydrophilic regions. We want to stress here, that we did not attempt to optimize the SAM quality, but merely exploit silane layers to affect the local wetting properties in enclosed channels.

Conducting a photolithography process inside a glass or quartz capillary tube is evidently more challenging than on a flat surface. Photoresist layers with strong adhesion onto the channel wall and uniform thickness are essential. Strong adhesion of these layers protects the desired pattern from the shear force during rinsing and chemical modification. A cleaned substrate with minimal surface water is desired to strengthen the adhesion of the photoresist layers. The presence of surface water can cause detachment of this layer during developing which destroys the desired pattern. ${ }^{22,23}$ Primers or adhesion promoters are typically applied onto cleaned glass or silicon substrates to exclude the surface water and enhance the adhesion. ${ }^{24}$ Silazanes and silanes, such as hexamethyldisilizane (HMDS), are typically used primers in cleanroom processes. However, such primers form covalent bonds to glass or silicon substrates and functionalize the complete channel surfaces which prevents the subsequent SAM pattern formation. Moreover, photoresist droplets may form on such surfaces after baking because of the low surface energy of the primer layers. Such thickness differences hamper the formation pattern structures since the developing rate is a function of the depth into the PR layers. ${ }^{25}$ In our case, an adequate vacuum baking step is essential to remove the surface water without the need for primers. A diluted base solution removes organic contaminants from the glass surface. We have prerinsed the surface with a TMAH solution, which is free of alkali metals. The optical images in Figure $3 a, b$ display the patterned capillary tubes after development. The smallest pattern we have fabricated was $60 \mu \mathrm{m}$ (Figure $3 \mathrm{~b}$ ). Because of the curved capillary geometry, the exposing UV light will be bend in the radial direction which should not reduce the axial edge sharpness. From microscopic observations we determined that the edge roughness is on the order of a few micrometers, which is close to the resolution of the optical mask we used.

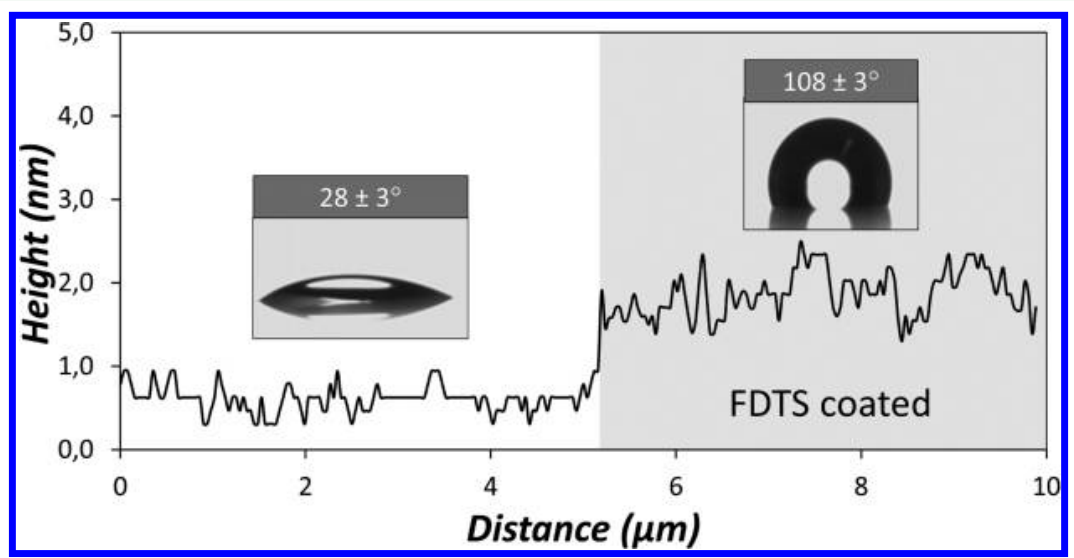

Figure 2. Height profiles of a modified flat glass sample measured by atomic force microscope and their corresponding contact angles. 


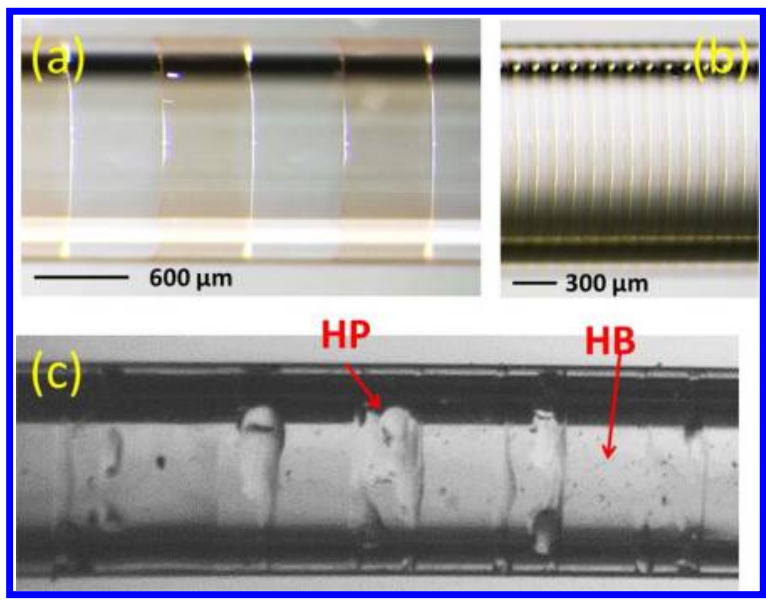

Figure 3. Patterned quartz capillary tubes after the photolithography. The yellow layer is the coated photoresist. The lengths of hydrophobic (HB) and hydrophilic (HP) segments are equivalent to (a) 600 and (b) $60 \mu \mathrm{m}$. (c) Water adhesion in a patterned quartz capillary tube.

Evidently, the edge roughness may be compromised when this method is applied in more complex microfluidic structures. Then also the photoresist coating can not rely on the spinning method employed here, but could be attempted using liquid displacement methods. As the fabrication is applied in a capillary tube, verifying the resulting surface wettability is challenging via contact angle measurements. We have attempted a facile method to test the modified tube by first filling it with DI water followed by gently removing the water with a nitrogen gas flow. Water remains on the hydrophilic segments but not on the hydrophobic segments (Figure 3c).

Additionally, we investigated the influence of the obtained heterogeneous surface wettability on altering the emulsion dynamics using a coflow microfluidic devices. The devices was built by carefully sliding a tapered micropipette into a modified glass capillary with inner diameter $d=580 \mu \mathrm{m}^{26}$ and an entrance hydrophobic section of $L=6 \mathrm{~mm}$. The oil-in-water emulsions were generated by injecting paraffin oil through the micropipette as inner phase and flowing aqueous solution (2 wt $\%$ PVA) through the glass capillary as continuous phase. The droplets were formed in various morphologies in the capillary by controlling the flow rates of the inner oil phase and outer aqueous phase via two corresponding syringe pumps. The dynamics of the segmented flows were visualized using a microscope (magnification $\approx 10$ ) equipped with a camera. Figure 4 shows the observed emulsion dynamics, when passing from a hydrophilic to a hydrophobic surface (highlighted in yellow). The heterogeneous wetting wall has a strong influence on the emulsion morphology. A fast moving droplet stays confined within the continuous phase when passing the heterogeneous wetting surface (Figure 4a). In contrast, droplets

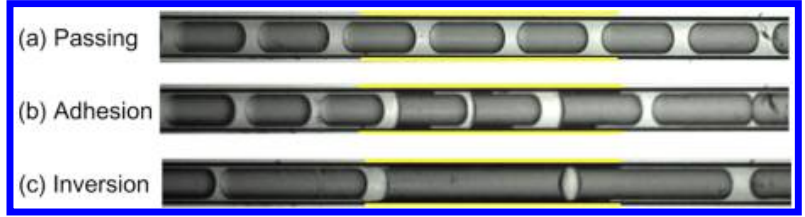

Figure 4. Effect of heterogeneous surface wettabilities on oil-in-water emulsions passing a hydrophobic section (marked in yellow, $L=6$ $\mathrm{mm}$ ) along a glass microcapillary. The velocities of the corresponding oil droplets are (a) 2.61 , (b) 0.27 , and (c) $0.61 \mathrm{~mm} / \mathrm{s}$. traveling at lower speed alter their emulsion dynamics in terms of adhesion (Figure 4b) and inversion (Figure 4c). It was reported that emulsion transitions can be used as a promising technology in biomedical and food processing. ${ }^{1,27-29}$ Our results give insight regarding the stability and dynamics of emulsions in confinement with heterogeneous wettabilities.

We have demonstrated positional patterning of surface wettability in a microcapillary tube. The method is highly reproducible and efficient as multicapillaries can be treated simultaneously. The modified microcapillaries display a heterogeneous surface with very sharp boundaries between the hydrophilic and hydrophobic segments. Our method can be employed to modify enclosed channel surfaces by various surface chemistries. The robust and inert characteristics of the SAM layer allows a secondary modification step on the initially protected hydrophilic surfaces. The smallest achieved dimension is $60 \mu \mathrm{m}$. This limitation is likely to be reduced by introducing a liquid with a similar refractive index as that of the capillary material to reduce the light scatting during exposure. Enclosed patterns have been demonstrated to influence the emulsion dynamics strongly, which is of interest in many applications.

\section{ASSOCIATED CONTENT}

\section{S Supporting Information}

The Supporting Information is available free of charge on the ACS Publications website at DOI: 10.1021/acsami.6b01842.

Experimental description on materials, detailed fabrication process, and AFM measurements (PDF)

\section{AUTHOR INFORMATION}

\section{Corresponding Author}

*E-mail: r.g.h.lammertink@utwente.nl. Phone: +31 53489 4798. Fax: +31534892882.

\section{Notes}

The authors declare no competing financial interest.

\section{ACKNOWLEDGMENTS}

The authors acknowledge the Dutch Technology Foundation STW (project 11396) for the financial support. We thank Dr. Cunlu Zhao for the AFM measurements and Manas $M$. Mandalahalli for the experimental assistance.

\section{REFERENCES}

(1) Shui, L.; van den Berg, A.; Eijkel, J. C. Interfacial Tension Controlled W/O and O/W 2-Phase Flows in Microchannel. Lab Chip 2009, 9, 795-801.

(2) Atencia, J.; Beebe, D. J. Controlled Microfluidic Interfaces. Nature 2005, 437, 648-655.

(3) Feng, Y.; Zhou, Z.; Ye, X.; Xiong, J. Passive Valves Based on Hydrophobic Microfluidics. Sens. Actuators, A 2003, 108, 138-143.

(4) Oh, K. W.; Ahn, C. H. A Review of Microvalves. I. Micromech. Microeng. 2006, 16, R13.

(5) Maruyama, T.; Matsushita, H.; Uchida, J.-i.; Kubota, F.; Kamiya, N.; Goto, M. Liquid Membrane Operations in a Microfluidic Device for Selective Separation of Metal Ions. Anal. Chem. 2004, 76, 44954500.

(6) Tetala, K. K.; Swarts, J. W.; Chen, B.; Janssen, A. E.; van Beek, T. A. A Three-Phase Microfluidic Chip for Rapid Sample Clean-Up of Alkaloids from Plant Extracts. Lab Chip 2009, 9, 2085-2092.

(7) Asanomi, Y.; Yamaguchi, H.; Miyazaki, M.; Maeda, H. EnzymeImmobilized Microfluidic Process Reactors. Molecules 2011, 16, 60416059. 
(8) Goto, M.; Tsukahara, T.; Sato, K.; Kitamori, T. Micro-and Nanometer-Scale Patterned Surface in a Microchannel for Cell Culture in Microfluidic Devices. Anal. Bioanal. Chem. 2008, 390, 817-823.

(9) Lopez, G. P.; Biebuyck, H. A.; Harter, R.; Kumar, A.; Whitesides, G. M. Fabrication and Imaging of Two-Dimensional Patterns of Proteins Adsorbed on Self-Assembled Monolayers by Scanning Electron Microscopy. L. Am. Chem. Soc. 1993, 115, 10774-10781.

(10) Feng, X.; Jiang, L. Design and Creation of Superwetting/ Antiwetting Surfaces. Adv. Mater. 2006, 18, 3063-3078.

(11) Kim, H.-C.; Wallraff, G.; Kreller, C. R.; Angelos, S.; Lee, V. Y.; Volksen, W.; Miller, R. D. Photopatterned Nanoporous Media. Nano Lett. 2004, 4, 1169-1174.

(12) Breisch, S.; de Heij, B.; Löhr, M.; Stelzle, M. Selective Chemical Surface Modification of Fluidic Microsystems and Characterization Studies. I. Micromech. Microeng. 2004, 14, 497.

(13) Priest, C. Surface Patterning of Bonded Microfluidic Channels. Biomicrofluidics 2010, 4, 032206.

(14) Yamamoto, M.; Yamada, M.; Nonaka, N.; Fukushima, S.; Yasuda, M.; Seki, M. Patterning Reactive Microdomains Inside Polydimethylsiloxane Microchannels by Trapping and Melting Functional Polymer Particles. I. Am. Chem. Soc. 2008, 130, 14044-14045.

(15) Kaji, H.; Hashimoto, M.; Nishizawa, M. On-Demand Patterning of Protein Matrixes Inside a Microfluidic Device. Anal. Chem. 2006, $78,5469-5473$.

(16) Vong, T.; ter Maat, J.; van Beek, T. A.; van Lagen, B.; Giesbers, M.; van Hest, J. C.; Zuilhof, H. Site-Specific Immobilization of DNA in Glass Microchannels via Photolithography? Langmuir 2009, 25, 13952-13958.

(17) O’Loughlin, M.; Priest, C.; Popescu, M. N.; Ralston, J. Patterning of wettability for controlling capillary-driven flow in closed channels. I. Colloid Interface Sci. 2013, 402, 259-266.

(18) Takei, G.; Nonogi, M.; Hibara, A.; Kitamori, T.; Kim, H.-B. Tuning microchannel wettability and fabrication of multiple-step Laplace valves. Lab Chip 2007, 7, 596.

(19) Chen, C.; Xu, P.; Li, X. Regioselective Patterning of Multiple SAMs and Applications in Surface-Guided Smart Microfluidics. $\underline{\text { ACS }}$ Appl. Mater. Interfaces 2014, 6, 21961-21969.

(20) Derzsi, L.; Jankowski, P.; Lisowski, W.; Garstecki, P. Hydrophilic Polycarbonate for Generation of Oil in Water Emulsions in Microfluidic Devices. Lab Chip 2011, 11, 1151-1156.

(21) Zhao, B.; Moore, J. S.; Beebe, D. J. Surface-Directed Liquid Flow inside Microchannels. Science 2001, 291, 1023-1026.

(22) Levinson, H. J. Principles of Lithographvi SPIE: Bellingham, WA, 2005.

(23) Glendinning, W. B.; Helbert, J. N. Handbook of VLSI Microlithography: Principles, Technology and Applications; William Andrew: Norwich, NY, 2012.

(24) Brewer, T.; Flaim, T. D.; Moss, M. G. Adhesion Promoting Product and Process for Treating an Integrated Circuit Substrate. US Patent US 4732 858, 1988.

(25) Dill, F. H.; Hornberger, W. P.; Hauge, P. S.; Shaw, J. M. Characterization of Positive Photoresist. IEEE Trans. Electron Devices 1975, 22, 445-452.

(26) Shah, R. K.; Shum, H. C.; Rowat, A. C.; Lee, D.; Agresti, J. J.; Utada, A. S.; Chu, L.; Kim, J.; Fernandez-Nieves, A.; Martinez, C. J. Designer Emulsions using Microfluidics. Mater. Todav 2008, 11, 1827.

(27) Hutton, C.; Campbell, A. Funcional Properties of a Soy Concentrate and a Soy Isolate in Simple Systems and in a Food System. I. Food Sci. 1977, 42, 457-460.

(28) Skurtys, O.; Aguilera, J. Applications of Microfluidic Devices in Food Engineering. Food Biophys. 2008, 3, 1-15.

(29) Chen, H.; Dong, E.; Li, J.; Stone, H. A. Adhesion of Moving Droplets in Microchannels. Appl. Phys. Lett. 2013, 103, 131605. 